\title{
THE HAZARDS OF BLOOD TRANSFUSION
}

\author{
By H. F. BREwer, M.D., B.Ch.(Cantab.) \\ Clinical Pathologist to St. Bartholomew's Hospital; \\ Medical Officer to the Greater London Red Cross Blood Transfusion Service
}

Blood transfusion used correctly is a life saving measure and its reputation and use have steadily increased since the 1939-45 war. Its growth and the present important role it plays in the practice of medicine have necessitated the State undertaking full responsibility for the provision of blood and blood products and of standard apparatus with which to collect and administer them. Last year the National Blood Transfusion Service distributed over 700,000 bottles of blood and in the London area the specialised Red Cross Service provided over 4000 donors purely for fresh blood transfusions. The spectacle of several patients in a Ward having simultaneous transfusions is a familiar one in the round of one's hospital duties. Nevertheless, in part owing to its ready availability, it is probable that a great deal of blood is given unnecessarily, and because blood can prove a lethal fluid and its administration carries certain risks, this aspect is particularly relevant in any discussion of the associated hazards. We have all seen blood given when the haemoglobin level has not really indicated it and where no account has been taken of the patient's own potential haemopoietic regenerative powers, especially when aided by generous diet, iron, folic acid or liver extract. Again, whole blood is sometimes transfused when plasma or serum or a plasma substitute (dextran) would achieve the same purpose or even be preferable. The indiscriminate use of blood by subjecting patients unnecessarily to some of the specific hazards is thus of itself a significant danger in blood transfusion and one that can only be eliminated by careful selection of the recipient and of the fluid to be administered. In the larger hospitals this is probably best assured by the appointment of a Transfusion Officer.

The hazards of blood transfusion may be listed as follows- -

(I) Haemolytic reactions dependent on intravascular haemolysis or on the transfusion of blood already lysed (extravascular haemolysis).

(2) Sensitization of the recipient.

(3) Use of the infected blood.
(4) Circulatory overloading.

(5) Air embolism.

(6) Transmission of disease from donor to recipient.

(7) Febrile and allergic reactions.

(8) Miscellaneous-e.g. Transfusion thrombophlebitis. Haemosiderosis.

This is a somewhat disturbing list and fortunate is the man who has had a long association with this form of treatment and has not seen it cause a fatality or harm a patient whom it was hoped to benefit.

\section{Haemolytic reactions}

As already indicated these may be due to the intravascular haemolysis of blood or to the transfusion of blood already lysed by some extravascular cause.

Intravascular haemolysis is the sequel of an incompatible transfusion and in most cases is dependent on the destruction of the donor's red cells by the action of specific iso-antibodies in the recipient's circulation. The destruction may be immediate or delayed, the severity of the haemolytic reaction running parallel with the rapidity of haemolysis. The significant antibodies concerned are anti-A and anti-B of the Landsteiner system and anti-D of the Rhesus system. Between them these are responsible for practically all the haemolytic reactions dependent on intravascular haemolysis of the donor cells. Anti-Kell is probably the only anti-body of the other group systems which has to be seriously considered.

Generally speaking an ABO incompatibility produces a reaction that is more acute and violent than one due to the Rhesus factor. With the latter the symptoms are usually (but not invariably) more silent and the appearance of jaundice after about 12 hours may be the first indication. A slow drip rate of administration is known to render the symptoms of incompatibility less obvious. They are also masked under general anaesthesia. Here, because no typical picture of incompatible transfusion is revealed, vigilance is particularly 
important. It is good practice to record the pulse rate and blood pressure in an anaesthetized patient at the commencement of a transfusion and on changing to a fresh bottle of blood, and then at 5 minute intervals for 15 minutes. If there is a sharp rise in pulse rate and/or a rapid fall in blood pressure, with perhaps some cyanosis, unexplained by any event or manipulation in the operation field, the transfusion should be terminated.

Of all the protean and well-known symptoms produced by the transfusion of incompatible blood severe lumbar pain is especially significant since it may be one of the earliest warnings of haemolytic reaction. If it occurs the transfusion should be discontinued until the possibility has been excluded by repetition of the grouping tests.

The development of reliable and sensitive techniques both for blood grouping and direct matching and the ready availability of potent grouping sera since the War have greatly reduced the incidence of incompatible transfusions due to imperfect serological methods.

The direct matching test between donor's red cells and the serum of the recipient is the final and essential precaution against incompatibility, and provided a satisfactory technique is employed it can be relied upon to detect not only $\mathrm{ABO}$ and $\mathrm{Rh}$ incompatibility but also any of the rarer antigen-antibody reactions, although their identity may not be known at the time. Performance of the direct matching test, which should never be omitted, carries a heavy responsibility, since it is almost the only laboratory investigation in which a mistake may prove fatal. In the writer's opinion it should for safety only be carried out by someone with laboratory training. For routine purposes a two tube technique in which donor cells in saline and in 20 per cent. bovine albumin are put up against the recipient's serum is very reliable and preferable to a tile method. The procedure is as follows-a few drops of donor blood are removed from the transfusion bottle (with sterile precautions) or from the pilot bottle and the cells washed twice with saline. A drop of 2-5 per cent. suspension in saline of these washed donor cells is placed in each of a pair of precipitin tubes with a Pasteur pipette. To both is added a similar sized drop of the patient's serum and mixing effected by tapping the tubes with the finger. One tube is placed in the incubator at $37^{\circ} \mathrm{C}$ and the other allowed to stand at room temperature. At the end of one and a half hours the supernatant saline in the incubated tube is cautiously pipetted off, discarded and replaced by a drop of 20 per cent. bovine albumin, without disturbing the underlying red cell sediment. The tube is put back in the incubator for a further half an hour. After this period the red cell deposit is removed from both tubes with a Pasteur pipette, gently spread on a slide, and examined for agglutinates under the low power of the microscope. In urgent circumstances a drop of a 2-5 per cent. suspension of washed donor cells in saline is placed in one precipitin tube and a drop of a similar strength suspension in 20 per cent. bovine albumin in another. To each is added a drop of the patient's serum. After tapping to mix, the tubes are placed in the $37^{\circ} \mathrm{C}$ incubator (or preferably a water bath) for at least 15 minutes. The tubes are then centrifuged just fast enough (I000 r.p.m. for one minute) to bring down the cells to the bottom of the tube. The sediment is then transferred to a slide and examined as described. Suspension of the donor cells in a protein medium is important in order to detect the presence of the so-called incomplete antibodies (mainly anti-D of the $\mathrm{Rh}$ system) which are a relatively common cause of potential incompatibility and which would be missed if a saline suspension alone were employed. The latter detects more effectively the antibodies anti-M, anti-N, anti-P anti-Lewis and non-specific cold agglutinins. For certain cases who are liable to have an immune isoantibody in their serum ( $\mathrm{Rh}$ antibody is the most significant)-sometimes referred to as 'dangerous recipients,' e.g.-Rh-negative patients, patientsog who have had previous transfusions or transfusion reactions, mothers of children where there is reason to suspect haemolytic disease, cases of acquired haemolytic anaemia-it is wise to augment the two tube test by the Indirect Coombs' test. This is more sensitive than the albumin technique in detecting traces of immune antibodies but it cannot alone be relied upon for demonstrating incompatibility dependent on naturallyoccurring anti-A and anti-B agglutinins.

Haemolytic reactions dependent on the destruction of the patient's own red cells caused by the transfusion of incompatible plasma, e.g. the use of a Group $\mathrm{O}$ donor for recipients other than Group $\mathrm{O}$, do occur. In most cases the concentration of the anti-A and anti-B agglutinins in the plasma of the Group $\mathrm{O}$ donor blood is diluted to a safe level in the recipient's circulation, but some Group $\mathrm{O}$ donors possess a high titre of these agglutinins, and particularly with massive transfusions given to a small and exsanguinated patient other than Group $\mathrm{O}$, can cause some intravascular haemolysis of the recipient's red cells. This seldom results in a frank haemolytic reaction comparable with destruction of incompatible donor cells and if the peace-time practice of only using homologous group ABO blood for a recipient is adhered to, a haemolytic reaction due to incompatibility of the plasma of the donor blood is extremely rare. It 
does not demand a direct matching test between donor's serum and recipient's cells. The responsibility of ear-marking Group $\mathrm{O}$ donors with high titre agglutinins should lie with the Transfusion Service. Bearing on the question of incompatible plasma is the production of anti-A and anti-B immune bodies in Group $\mathrm{O}$ individuals following the injection of horse serum and prophylactic bacterial vaccines. Such immune antibodies are capable of reacting powerfully with A or B cells. Safety lies in ensuring that a Group $\mathrm{O}$ donor who has had for example anti-tetanus serum or prophylactic TAB vaccine in recent months is used only for Group $\mathrm{O}$ recipients and not as a 'universal' donor.

Extravascular haemolysis. Haemolytic reactions due to massive extravascular destruction of the donor red cells may result from the use of timeexpired stored blood, and of blood that has been accidentally overheated or frozen.

Haemolytic reactions whether due to intravascular or to extravascular haemolysis are usually the result of gross blunders. Blood of an incompatible group is given because of a clerical error, or confusion between patients with similar names. Haste in blood grouping and the direct matching test-or omission of the test-are much more significant causes than the action of obscure antibodies. In most hospitals time and money are better spent on improving transfusion organization rather than on the development of elaborate compatibility techniques-provided that homologous $A B O$ and $R h$ blood is selected, and a reliable direct matching test, such as that already described, is performed. An additional label put over the cap of a bottle of direct matched blood and bearing the caption' Stop ! Have you checked full name of patient and Ward' on the compatibility label is a wise injunction. If blood has to pass through several hands before being issued for and given to a particular patient its identity should be checked en route in the same way as a dangerous drug. Even in urgent cases, the initial use of a plasma substitute will practically always tide the patient over until grouping tests can be completed. Collapse is due more to lack of blood volume than lack of red cells.

Nursing staff should be told of the danger of overheating blood; they seldom realize that blood is damaged by exposure to temperatures above $38^{\circ} \mathrm{C}$. It is a wise rule for anyone carrying out transfusions not to allow a bottle of blood to be immersed in warm water unless the temperature of the water has been first assessed by his own hand. In practice, with certain exceptions-e.g. a high titre of cold agglutinins-preliminary warming of the blood when given at a slow drip rate is unnecessary.
Blood which has thawed after being frozen is equally dangerous. Such blood usually shows obvious haemolysis to the naked eye. Accidental freezing is avoided by fixing the thermostat controlling the temperature of the storage refrigerator to a range between $2^{\circ}-4^{\circ} \mathrm{C}$ and by charting the daily records of a maximum and minimum thermometer placed in it. Generally speaking domestic refrigerators are not satisfactory for storing blood since temperatures vary from one part of the cabinet to another.

Before concluding the subject of haemolytic transfusion reactions there is now some comfort to be derived from the fact that even in severe examples, when anuria has developed, the regime advocated by Bull and his colleagues has proved to be a great advance in reducing the mortality, previously associated with these disasters. Essentially this treatment (which aims at resting the kidney as much as possible during the stage of impaired function) consists of a protein-free, mineral-free diet containing an adequate number of calories and having a water content of only 1000 c.c. per day (corresponding to the amount lost via the lungs, skin and faeces). It is administered, owing to its unpalatability (an emulsion of glucose and arachis oil) through a permanently indwelling stomach tube at a drip rate and continued until the patient commences to pass 1000 c.c. of urine a day. This treatment which should be started at once is the only one which has so farot improved the prognosis in the anuric patient; provided the kidneys were previously healthy it yields a high incidence of recoveries. If it cannot be instituted where the patient has had the transfusion, he should be transferred to a hospital with experience of it.

Administration of alkalis and attempts to flush the kidney by pushing fluids at this stage are harmful and to be deprecated.

\section{Sensitization of the recipient}

The danger here chiefly relates to the $\mathrm{Rh}$ antigen. A single transfusion of $\mathrm{Rh}$-positive blood given to an $\mathrm{Rh}$-negative recipient may result in sensitization to this antigen and, even if no obvious immediate harm results, may be a potential source of danger with subsequent transfusions.

In an Rh-negative woman, such sensitization by $\mathrm{Rh}$ positive blood is particularly to be avoided since indirectly it may have a devastating effect on future foetuses should she marry an Rh-positive male. Not only are her infants more likely to be afflicted by haemolytic disease-and of a severe type-but the first child, which usually escapes when the immunization is due to pregnancy alone is frequently diseased and may not survive. It is 
thus extremely important to ascertain prior to transfusion the $\mathrm{Rh}$-group of any female patient in or before the child-bearing period of life, and only to administer Rh-negative blood should she prove to be in this category. Taking any chance on this amounts to negligence. The counsel of perfection, of course, is to $\mathrm{Rh}$ group all recipients and to use only homologous $\mathrm{Rh}$ blood.

Repeated transfusions carry an enhanced risk of sensitisation and the production of antibodies to some of the rarer antigens. Certain patients such as those with acquired haemolytic anaemia are especially liable. As already indicated an indirect Coombs' test should be included in the direct matching in these cases.

\section{Infected blood}

Infected blood is a hazard of stored blood, since freshly drawn blood is bactericidal and with a fresh blood transfusion the blood is given before sufficient time can elapse to allow of serious multiplication of any contaminating organisms. The bactericidal property of fresh blood, however, rapidly diminishes with refrigeration and storage, and stored blood becomes a good culture medium. It may even be to advantage to avoid refrigeration of freshly drawn blood for a few hours after collection and so enable it to overcome possible stray contaminants.

Contamination may occur in many ways-from the skin of the donor at the time of bleeding (even blood cultures taken under ideal conditions have a small but definite contamination rate with skin organisms), from the use of imperfectly sterilized bottles, collecting sets or anticoagulant diluent, dependent on inefficient autoclaving, and by the introduction of organisms when the bottle of blood is opened for the preparation of 'packed cells.' The use of a pilot bottle has obviated the necessity of entering the main bottle to obtain a blood sample for the direct matching test and has eliminated at least one possible source of contamination. Any infection, however introduced, is likely in stored blood to result in a heavy bacterial growth if the blood is allowed to remain at room temperature for a few hours. Relating to this, the practice of having several bottles of blood, which have been direct matched for a particular patient, standing by in the theatre during an operation until they are required, is to be condemned. Blood in reserve should be kept in the refrigerator until just before use.

The transfusion of even a small volume of grossly infected blood can produce a condition akin to severe protein shock, with circulatory failure and rapid death. Such blood, dependent on the type of organism present, may or may not show to naked-eye inspection haemolysis or a pigment change, either of which should raise suspicion. There may also be a faint smell of hydrogen sulphide on opening the bottle. Atypical coliform bacilli capable of multiplying at refrigerator temperature have proved to be the most significant organisms. When present in large numbers an infecting organism may be demonstrable in a direct smear made from the residual blood in the bottle or from the heart's blood of the patient post-mortem. Having in mind the risks to sterility to which stored blood may be exposed, it is gratifying that fatalities from this hazard are so few, but it is possible that minor degrees of bacterial contamination are responsible for some febrile reactions.

\section{Circulatory overloading}

This is related not to the properties of the transfused blood but to the total volume given and the speed of administration. It is a transfusion danger when the condition of the myocardium is impaired as the result of severe chronic anaemia, cachexia or coronary disease. In such circumstances any considerable increase in venous pressure may precipitate cardiac failure, and when transfusion is indicated, the aim must be to introduce red cells without producing any appreciable rise in venous pressure. Overloading in addition to raising venous pressure results in an increase in the amount of blood in the pulmonary circulation, and a diminution in vital capacity. Prophylaxis consists in giving the blood at a very slow drip rate (0.5-I c.c. per pound of bodyweight per hour), in limiting the volume at one transfusion to less than 500 c.c., in the use of packed cells which, compared with whole blood, achieve the same rise in haemoglobin level for half the volume and finally in warming the patient. This last procedure, by causing dilatation of the blood vessels in the skin and limbs, tends to diminish the amount of blood in the pulmonary circulation, where at least part of the increased blood volume resulting from too rapid a transfusion is normally accommodated. It also helps to avoid a rigor which would put an additional burden on the heart. Symptoms and signs which give warning of overloading are-tightness in the chest, a dry cough, dyspnoea, cyanosis, engorgement of the cervical veins, and an increased pulse rate. Their appearance, if unheeded, is followed by pulmonary oedema with moist sounds at the lung bases and then rapid circulatory failure. Treatment consists in stopping the transfusion, propping the patient up and giving atropin and a cardiac stimulant by hypodermic injection.

\section{Air embolism}

About ro c.c. of air introduced into the circula- 
tion of a sick person may prove fatal. Such an accident may be due either to faulty technique or to faulty apparatus. The risk is greatest when positive air pressure, e.g. via a Higginson's syringe is employed to drive blood into the constricted veins of a patient with oligaemic shock, or when an arterial transfusion is being carried out. A procedure of this nature should be in the hands of a responsible person lest the bottle become empty unnoticed.

Leaks in perished rubber tubing of the standard administration set may allow air to be sucked into the drip chamber if the screw clip controlling the rate of flow is placed above the chamber. To avoid this, the clip should be located as low down as possible. Other precautions against air embolism are ensuring that air is driven out of the tubing of the administration set by running blood through it before commencing a transfusion (the needle end of the set being held on a level with the bottle), and also changing to another bottle before the preceding one has quite emptied so that the tubing is kept full of blood.

\section{Transmissible diseases}

There are three diseases of practical importance which may be transmitted-hepatitis, malaria and syphilis. The danger of virus hepatitis has been chiefly associated with the transfusion of pooled human plasma or serum. The incubation period is long-80-I20 days, and the severity very variable. There may be mild transient jaundice with little inconvenience or massive hepatic necrosis with a fatal termination. In the past the incidence following the transfusion of these blood products was as high as io per cent., but a reduction in the number of donors contributing to a pool to 8-io has greatly lowered the frequency and the current incidence is about I per cent. Even so this is still much higher than that of any other infection transmitted by transfusion and there is much to be said for the use of a plasma substitute (dextran is the most physiological of those available) in place of plasma or serum until some effective method of killing the virus is discovered. The virus survives drying and neither irradiation with ultra-violet light nor treatment of the plasma or serum with nitrogen mustard-two lines of eradication attempted - appear capable of achieving this. The risk with whole blood is small except with repeated transfusions or when a single large volume transfusion entailing many donors is given (the hazard of hepatitis from ro bottles of blood approximates to I of 'small pool' plasma or serum).

Some years ago it was customary for prophylaxis to exclude a volunteer from giving blood for a period of 12 months after a bout of hepatitis.
Recent work has demonstrated that the virus may persist in the blood in a ' carrier' state for many years after the original attack, and it is now considered safer not to accept any prospective $\frac{\alpha}{\infty}$ donor giving a history of infective hepatitis, nor $\stackrel{\varrho}{c}$ anyone who has been a contact with the disease within six months. Unrecognized sub-clinical $\stackrel{\vec{S}}{\rightarrow}$ grades of mild jaundice in donors are undoubtedly a potent source of infection. There is as yet no $\overline{0}$ easily performed laboratory test capable of $\frac{\bar{\omega}}{\bar{\sigma}}$ revealing the presence of the virus.

The problem of malaria is one mainly relating to malarial endemic centres but it is of some significance in this country with the numerous $\vec{\circ}$ ex-Service men who have been in such areas and $\overrightarrow{\vec{\omega}}$ with the present ease and facilities for foreign $\stackrel{\omega}{\omega}$ travel. Observations bearing on this hazard are $\overrightarrow{0}$ that malarial parasites can survive in stored blood 3 . refrigerated at $2^{\circ}-4^{\circ} \mathrm{C}$ throughout its period of usage (i.e. up to 3 weeks from the date of collec- $\omega$ tion); also, that even when a donor has not resided ${ }_{\infty}$ in a malarious country for a very long time, his is blood may still harbour parasites, particularly in 0 the case of a quartan infection. With the efficacy of modern chemotherapy, the chances of an individual transmitting this disease who has had $c$ malaria five years or more previously, and who has not been exposed to the infection since, nor $\vec{\theta}$ had any febrile bouts that could be attributable of it is extremely small.

Transfusion syphilis is now in Great Britain very remote risk and still receding. Factors contributing to this are the low incidence of the disease, the efficacy of modern treatment and $\stackrel{\circ}{\circ}$ the inability of the spirochaeta pallida to survive $\unrhd$ for more than 72 hours in blood stored at $2-4^{\circ} \mathrm{C}$. $\overrightarrow{\overrightarrow{0}}$ The limitations of a serological test, such as a 3 Wasserman reaction, as a safeguard with donor blood must be appreciated; the late primary or early secondary stages of syphilis, times when the 음 serological test may be negative or at least doubtful, 3 . are the most infectious periods, whereas the $\frac{\sigma}{3}$ chronic tertiary in whom the result is strongly positive is probably quite innocuous.

\section{Febrile and allergic reactions}

Febrile reactions. It is difficult to assess the true $D$ incidence of febrile reactions associated with blood transfusion because figures quoted usually give $\mathrm{N}$ no information about the frequency with which the temperature of the patient was recorded, nor 0 over what period after the transfusion. In the $\omega$ writer's experience if the temperature is taken half hourly during a transfusion and for at least 20 hours afterwards few febrile reactions will be $\mathbb{\varnothing}$ overlooked and the incidence of fever up to $100^{\circ} \mathrm{F}+$ is approximately i 5 per cent. About 5 per cent. $\frac{0}{0}$ of patients have a greater rise which may be $\frac{\vec{D}}{\mathbb{D}}$ 
accompanied by a rigor. When a febrile reaction develops it usually does so within 90 minutes of the end of a rapid transfusion or during the course of a slow transfusion. Although the fever may extend to the next and following days it is most unlikely for a febrile reaction of this nature to commence on the day after the transfusion. In such a case a further cause must be sought.

Sterile compatible blood can produce a febrile reaction but there is no doubt that the chief cause is the presence in the anticoagulant fluid or the apparatus of foreign protein and polysaccharidesderived from dead bacteria, disintegrated or intact, and from the products of their growth. The incidence of febrile reactions can be reduced to a low incidence by adopting scrupulous care in the preparation of anticoagulant solutions and in the cleaning of used apparatus as follows-

I. The distilled water should be prepared in all-glass or specially designed stills.

2. All solutions must be prepared from freshly distilled water (previous few hours) and not from water distilled on the previous day.

3. The solutions should be autoclaved as soon as they have been prepared.

4. The still itself and the bottles into which the solutions are to be put must be rinsed with freshly distilled water.

5. Used or new rubber tubing and glassware must be cleansed thoroughly so as to remove all debris and they should be finally rinsed in distilled water and then dried rapidly.

The introduction of expendable sets which are discarded after use would be an advantage.

The risk of febrile reactions is also lessened by

(a) Giving the transfusion slowly e.g. a pint in 3-4 hours.

(b) Keeping the patient warm, or even hot, throughout the transfusion-e.g. well wrapped, hot water bottles, windows closed to exclude draughts. This warming up as previously mentioned also reduces the risk of circulatory overloading.

(c) In a nervous or already febrile patient the administration of an opiate and/or an antihistamine drug before commencing the transfusion.

A special point bearing on febrile reactions in infants is worth emphasizing. It is that infants seem incapable of shivering. They often, however, with a febrile reaction exhibit pallor and have a cold skin, also accompanied by a refusal to feed and by some diarrhoea.

Allergic reactions. These are recognised by the appearance of a rash, swelling of mucous membranes, urticarial weals, itching and at times nausea and vomiting. Asthma is rare and may be difficult to distinguish from the results of circulatory overloading. Occasionally there may be a rise of temperature. In most patients there is a history of some allergic affection. These reactions are usually associated with the donor plasma component of the transfusion and are due to the release of histamine acting on the receptor's cells. Severe cases resemble shock and the patient shows marked apprehension. Prophylaxis in a known allergic recipient may be achieved by the giving intramuscularly of one of the antihistamine drugs-antozoline hydrochloride (antistin) roo mg. or promethazine hydrochloride (phenergan) 25 mg. just before the transfusion. The addition of chlorprophenpyridamine maleate (piriton) $10 \mathrm{mg}$. to the transfusion bottle immediately prior to administration of the blood has also proved protective. Packed cells (in which the amount of plasma is minimal) or saline washed cells may be used with success for patients known to be allergic to plasma. For treatment-adrenalin hydrochloride (I-1000), ro minims, or ephedrine hydrochloride, $\frac{3}{4}$ grain, by hypodermic injection are indicated. Sensitivity can be passively transferred from an allergic donor to a patient, particularly if the donor's allergy is active at the time of bleeding. Many specific examples are now on record. The moral is to reject as blood donors those who give a history of hypersensitivity.

\section{Miscellaneous hazards}

Transfusion thrombophlebitis is a not infrequent complication of prolonged blood transfusions but more especially of saline and glucose infusions. Its incidence seems to be very variable in hospitals in different parts of the country. The condition can prove painful to the patient and also seriously interfere with intravenous post-operative fluid therapy. The red rubber tubing used in the standard transfusion administration sets has been suspect and certainly latex rubber tubing appears less liable in the hands of some operators to cause trouble. It is possible that a substance noxious to the vein wall is gradually extracted from the red rubber tubing by the fluid passing through itbut this is by no means certain. Other factors such as rough handling of the vein may play a part. It is probably a wise precaution to employ a latex tubing set for a transfusion likely to exceed 18 hours and in the case of a saline and/or glucose infusion to change the needle to another vein after it has run for this period.

Periodic blood transfusions may be lifesustaining for patients with a refractory, chronic haemolytic, or aplastic anaemia, but the necessity of such regular administration of blood is liable in time to result in haemosiderosis. Since every pint of blood carries into the patient's body 
approximately $250 \mathrm{mg}$. of iron and since there is no pathway for excretion of iron, the giving of large numbers of blood transfusions gradually leads to the deposition in various organs of the body of considerable quantities of iron which may in time interfere with their functions. Haemosiderosis may affect the bone marrow, myocardium, kidneys and adrenal glands. The liver may become cirrhotic or diabetes may result from siderosis of the pancreas.

In conclusion every blood transfusion, even in experienced hands, carries certain hazards and it is important that before a clinician recommends this ‡ particular therapy he should carefully weigh up for 3 each individual patient the advantages to be gained $\stackrel{\mathbb{Q}}{\mathcal{C}}$ and balance them against the potential dangers $c$. and ill effects.

\section{References}

Bull, G.M., Joekes,A.M., and Lowe K.G. (1949), Lancet, 2. 220, Discombe, G. (1955), “ Blood Transfusion", Heinemann, London Keynes, G., (1949), “ Blood Transfusion”, Wright, Bristol. Mollison, P.L. (1951), "Blood Transfusion in Clinical Medicine".

\section{PULMONARY TUBERCULOSIS \\ (Ṕostgraduate Medical Journal)}

Price 3s. 10d. post free

INTRODUCTION

Maurice Davidson, D.M., F.R.C.P.

THE GENERAL MANAGEMENT OF THE PHTHISICAL PATIENT FROM THE PHYSICIAN'S STANDPOINT

L. E. Houghton, M.D.

THE PRESENT POSITION OF CHEMOTHERAPY IN TUBERCULOSIS

John C. Roberts, M.D., M.R.C.P.

THE PROBLEM OF TUBERCULOSIS IN CHILDHOOD

A. Margaret Macpherson, M.D., F.R.C.P.
COLLAPSE THERAPY (MEDICAL) IN PULMONARY TUBERCULOSIS

F. H. Scadding, M.D., M.R.C.P.

SURGICAL COLLAPSE THERAPY IN PULMONARY TUBERCULOSIS

J. R. Belcher

RESECTION FOR PULMONARY TUBERCULOSIS

W. P. Cleland, M.R.C.P., F.R.C.S.

MODERN METHODS OF DIAGNOSIS IN PULMONARY TUBERCULOSIS: CLINICAL, BACTERIOLOGICAL AND RADIOLOGICAL

J. R. Bignall, M.D., M.R.C.P.

Published by

THE FELLOWSHIP OF POSTGRADUATE MEDIChNE

60, Portland Place, London, W.1

\section{Index for 1955 is now ready. Have your Journals bound up into a volume NOW. See advertise- ment on page 296 for details.}

